\title{
Comparison of bilateral medial rectus recession vs recession-resection as surgery for infantile esotropia
}

\author{
Aman Kumar Gupta ${ }^{1}$, Kabindra Bajracharya ${ }^{2}$, Roshan Dev Yadav ${ }^{3}$, Salma K.C. Rai ${ }^{4}$ \\ ${ }^{1,3}$ Opthalmologist, ${ }^{2}$ Associate Professor and Consultant Pediatric Ophthalmologist, Lumbini Eye Institute and \\ Research Center, Bhairahawa, Nepal. ${ }^{4}$ Professor and Consultant Pediatric Ophthalmologist, Shree Krishna Natralaya, \\ Bhairahawa, Nepal.
}

Background: Infantile esotropia is misalignment of eyes from bifoveal fixation where surgical correction at an early age usually presents with good outcomes. Aims and Objective: To compare the surgical outcome between bilateral medial rectus recession (BMR) and recessionresection (RR) surgery in infantile esotropia. Materials and Methods: The study was conducted in Lumbini Eye Institute, Bhairahawa, from December 2017 to December 2018. Patients with infantile esotropia who underwent surgical correction were included in the study. Patients were examined pre operatively, and post operatively at day one and third month, where the angle of deviation was measured and the level of binocular single vision (BSV) was assessed. Data was entered and analyzed using the SPSS program. Results: Total 79 patients were included in the study out of which 33 underwent BMR and 46 underwent RR. The mean preoperative angle by PBCT was 52.58 PD (SD \pm 15.468 ) in the BMR group and 47.83 PD (SD \pm 12.140 ) in the RR group. Post-operative deviation in the BMR group had angle <35 PD. In the RR group the post-operative deviation observed was $<35 \mathrm{PD}$, whereas $1 / 46$ patients had angle between 35-50 PD. All patients who had residual esotropia (ET) in either the BMR or RR group had a pre-operative angle above 50 PD. Pre-operatively $43 \%$ had fusion and rest of them had suppression. Post-operatively $40.5 \%$ had fusion, $15.2 \%$ had stereopsis and rest of them had suppression or BSV could not be assessed in them. Therefore, there was increase in BSV by $2.5 \%$ from fusion to stereopsis. Those who initially showed suppression or in whom the BSV could not be assessed pre-operatively now showed to have fusion or some stereopsis. In total there was $15.2 \%$ increase in stereopsis. Conclusion: Though cosmesis has been improved in most patients after strabismus surgery, BSV improvement was insignificant as most surgery was performed after attainment of visual maturity. No significant difference between BMR and RR was found in terms of improvement of angle of deviation and BSV.

\section{Access this article online}

Website:

http://nepjol.info/index.php/AJMS DOI: 10.3126/ajms.v12i8.36171

E-ISSN: 2091-0576

P-ISSN: $2467-9100$

Copyright (c) 2021 Asian Journal of Medical Sciences

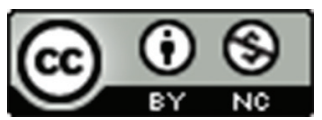

This work is licensed under a Creative Commons Attribution-NonCommercial 4.0 International License.

Keywords: Infantile esotropia; Bilateral Medial Rectus Recession (BMR); Recession-

Resection (RR); Binocular Single Vision

\section{INTRODUCTION}

Strabismus (squint) is a misalignment of the eyes in which the visual axes deviate from bifoveal fixation. ${ }^{1}$ It can be subdivided into esotropia (inward deviation), exotropia (outward deviation) or, less commonly, hypertropia (upward deviation), hypotropia (downward deviation) and cyclotropia (torsional deviations - inwards, incyclotorsion or out outwards, excyclotorsion). This study specifically looked at infantile esotropia (IE). Other terms that have been used to describe this condition include congenital esotropia and essential infantile esotropia. Strabismus is present in approximately $4 \%$ of children. ${ }^{2}$ The reported incidence of infantile esotropia within the first six months of life varies between $0.1 \%$ and $1 \% .^{3}$

Infantile esotropia is a large, constant, stable angle esotropia (the angle indicates the degree/size of the squint) with an 
onset within the first six months of life. Features associated with IE include: alternating esotropia (can swap from one eye to the other); cross-fixation: taking advantage of the crossed position of the eyes so that the right eye is used to look towards the left and the left eye to look towards the right. This can be associated with the appearance of limited abduction (outward movement) of the other eye; manifestlatent nystagmus: oscillation of the eyes, which increases when either eye is covered; asymmetry of optokinetic nystagmus: a following movement followed by a rapid fixation movement in the opposite direction. ${ }^{1}$ The main reason for presentation of children with infantile esotropia is usually parental awareness of unacceptable ocular misalignment.

Treatment aims to improve ocular misalignment. However, another important issue to consider is whether such treatment facilitates and enhances the development of binocular vision. Therefore, there are two aims of intervention: (1) to align the visual axis, (2) to optimise the potential for binocularity. Many authorities believe that alignment to within 10 dioptres $\left.{ }^{\wedge}\right)$ of orthotropia 'straight eyes' by two years of age offers the best prospect for the development of binocular vision. ${ }^{4}$

Management of the strabismus may be surgical, non-surgical or a combination of both. Surgery to correct the esotropia involves adjusting the horizontally acting extraocular muscles, and can be divided into three types: (1) Unilateral surgery: weakening, usually recession, of the medial rectus which is responsible for pulling the eye in, and resection (strengthening) of the lateral rectus which is responsible for pulling the eye out, (2) bilateral surgery: the medial rectus is weakened in both eyes; three or more muscle surgery (horizontal): a combination of recessions and resections.

Surgical adjustment of the vertically acting muscles may also be undertaken to correct any significant hypertropia: (1) weakening of the inferior oblique muscle responsible for pulling the eye up in adduction; (2) weakening of the superior rectus, responsible for pulling the eye up in abduction, adduction and in the primary (straight ahead) position.

The age at which surgery is performed can vary and authors have used various terms to describe the timing of surgery. For example, 'ultra-early' has been used to describe surgical intervention between four and six months, 'early' to describe surgery before the age of two, and 'late' to describe surgery after the age of two. ${ }^{1}$

The main form of non-surgical management in IE is botulinum toxin. The toxin is injected into the medial recti to temporarily paralyze the muscles and weaken their action, allowing the antagonist muscles (the lateral recti) to act unopposed. When the paralytic effect wears off after several months, the alignment may be improved. ${ }^{5}$
Infantile esotropia (prevalence $0.2-0.35 \%$ ) is usually corrected by posterior relocation of the insertion of the medial rectus of both eyes (bilateral recession, BR) or unilateral recession of the medial rectus muscle combined with a resection of part of the tendon of the lateral rectus muscle (recession-resection, RR). A good surgical result is a slight esotropia, because overcorrection into exotropia may lead to double vision. Complete restoration of binocular vision or stereopsis is rare. To date, there have been no randomized controlled trials to compare BR with RR. In a recent Cochrane review on interventions for infantile esotropia it was concluded that the preference of BR or RR is subject of discussion and further research was necessary.

\section{MATERIALS AND METHODS}

The research was a hospital based, controlled, randomized study and children attending the Pediatric Ophthalmology and Strabismus Department of Lumbini Eye Institute, were part of this study. Approval was obtained from Institutional Review Board (IRB), and the research was conducted only after obtaining the clearance from IRB. Written and informed consent was obtained from both parents of the children, and one parent in case of single parenthood. Inclusion of study subjects occurred over a period of 12 months. Sample size was calculated based on the hospital records of the patient attending the strabismus clinic. Patients were randomly assigned in two groups by lottery system. Inclusion criteria was: (1) all children aged less than 15 years with a history of esotropia before with a normal developmental milestone. Exclusion criteria was: (1) Patients with unreliable follow up (2) Any esotropia other than infantile esotropia were excluded (3) Subject not willing to give their consent or not willing to participate in the study were excluded (4) Patient previously operated or undergone previous squint surgery.

A complete ophthalmic history was recorded using a detailed history. Specially designed proforma was used for the study. Demography of the patient including age, sex, address, telephone number, ethnicity, was obtained. The preoperative orthoptic examination consisted of the following procedures: refractive error was determined by retinoscopy and fully corrected before determining the angle of strabismus. If present, amblyopia was treated before entry in the study. The measurement of the manifest and latent angle of strabismus was performed by prism cover- un-cover test and alternating prism cover test at near with a fixation object, and at distance with a fixation light. After the final preoperative examination, the child was randomly assignment by lottery system to either BR or RR. Postoperatively, two orthoptic examinations was performed: 1 . immediately after the bandage was taken off on the day after surgery, the angle of strabismus was 
estimated by observing the corneal light reflex at one meter distance; 2 .at three months, an extensive orthoptic examination was performed, and was used for final analysis.

\section{Surgical Techniques}

The consensus surgical protocol contained regulations for the surgical plan and for the surgical technique. It prescribes that the total relocation of the two muscles, in millimeters; either BMR or RR was calculated by Park's criteria. Pediatric ophthalmologists and strabismologist having a minimum of two years of experience with both recessions and resections performed the surgery.

\section{Data Analysis}

Data pertaining to patients' demographics, history, signs, investigations report and causes of visual morbidity were filled up in the proforma specially designed for the study. The collected data was checked and coded manually and then entered into the computer. The numerical data obtained from the study was analyzed by SPSS version 16 . Data was expressed in frequency, percentage, mean and standard deviation as applicable.

\section{RESULTS}

Total of 79 patients were included in the study with no loss of follow up. Thirty-three out of 79 patients underwent BMR and 46 underwent RR. The BMR group had a mean age of 7.15 years ( $\mathrm{SD} \pm 4.147$ ); while 46 patients in the $\mathrm{RR}$ group had a mean age of 11.19 years ( $\mathrm{SD} \pm 4.736$ ); with 16 females and 17 male candidates in BMR group; while the RR group consisted of 25 female and 21 males. The mean pre-operative angle by Hirsberg test in the BMR group was 22.88 degree ( $\mathrm{SD} \pm 8.294)$, whereas the RR group had a mean angle of 20.87 degree (SD \pm 7.326 ). The mean preoperative angle by PBCT was $52.58 \mathrm{PD}$ (SD \pm 15.468$)$ in the BMR group and 47.83 PD (SD \pm 12.140$)$ in the RR group. The mean age when squinting was first noticed in the BMR group was 2.52 years ( $\mathrm{SD} \pm 1.004$ ), while in the $R R$ group it was 2.65 years $(\mathrm{SD} \pm 1.337$ ). Out of 79 patients 22 of them underwent patching before surgery (27.8\%). Out of 22 patients who underwent patching, 9 of them were of BMR group (40.90\%) and 13 of them were of RR group (59.09\%). Out of 22 patients who underwent patching 8 of them had fusion, 11 of them had no fusion while in 3 of them the level of BSV could not be assessed. Forty-two were Nepali nationals $(53.2 \%)$ while 37 were Indian nationals (46.8\%). Fifty-four (68.4\%) patients had intermittent esotropia while 25/79 (31.6\%) had constant esotropia. Only $2 / 79(2.5 \%)$ had a positive family history of squinting. $29 / 79(36.7 \%)$ patients had been using glasses before surgery while 50/79 (63.3\%) did not wear glasses.
Force duction test (FDT) was negative in all who underwent surgery for infantile esotropia. On comparing the level of binocular single vision (BSV) pre-operatively (Table 1) and post-operatively (Table 2) at the 3 months of follow up, it was seen that $17 / 33$ patients in the BMR group had fusion, $8 / 33$ did not have any BSV while in the rest $8 / 33$ the BSV could not be assessed. Post-operatively during the second follow up at third month $8 / 33$ developed stereopsis (Table 2). It was seen that 4 out of the 17 candidates in the fusion group now developed stereopsis post-operatively during their second follow up at the third month. One of the candidates who did not have any level of BSV now developed stereopsis after surgery; when in 3 out of the 8 candidates in whom the BSV could not be assessed now showed to have stereopsis. In the RR group, pre-operatively 17/46 had fusion, 28/46 did not have BSV, and in 1/46 BSV could not be assessed (Table 1). Post-operatively during the second follow-up at third month 4 of them in the RR group developed stereopsis, where as 2 additional candidates developed fusion, with a total of 19 candidates in the fusion group post operatively compared to 17 in the preoperative period. In 5 of 28 candidates who had no BSV pre-operatively, now developed either fusion or stereopsis.

The angle of the squinting eye was measured by prism bar cover test (PBCT) pre-operatively (Table 3) and post-operatively (Table 4) during the second follow up at third month. In the BMR group 6/33 patients had angle $<35 \mathrm{PD}, 8 / 33$ had angle ranging between 35-50 PD, and $19 / 33$ had an angle $>50 \mathrm{PD}$. Post-operative angle in the BMR group showed all 33 patients now had angle $<35$ PD. Among these 33 patients 28 of them had no angle post-operatively, 3 of them had a residual ET of $10 \mathrm{PD}, 1$

\begin{tabular}{|c|c|c|c|c|}
\hline & & \multicolumn{2}{|c|}{ Surgery type } & \multirow[t]{2}{*}{ Total } \\
\hline & & BMR & $\mathbf{R} / \mathbf{R}$ & \\
\hline Pre-op & Fusion & 17 & 17 & 34 \\
\hline level of & None & 8 & 28 & 36 \\
\hline BSV & could not be assessed & 8 & 1 & 9 \\
\hline Total & & 33 & 46 & 79 \\
\hline
\end{tabular}

BSV- Binocular Single Vision; BMR- Bimedial Recession; RR- Recession-Resection

\begin{tabular}{llccc}
$\begin{array}{l}\text { Table 2: level of BSV versus surgery type } \\
\text { post-operatively }\end{array}$ & \\
\hline & & \multicolumn{2}{c}{ SURGERY TYPE } & Total \\
\cline { 3 - 4 } & & BMR & R/R & \\
\hline Post-op & Fusion & 13 & 19 & 32 \\
level of & Stereopsis & 8 & 4 & 12 \\
BSV & None & 7 & 23 & 30 \\
& could not be assessed & 5 & 0 & 5 \\
Total & & 33 & 46 & 79 \\
\hline BSV-Binocular Single Vision; BMR- Bimedial Recession; RR- Recession-Resection
\end{tabular}




\begin{tabular}{|c|c|c|c|c|}
\hline & & \multicolumn{2}{|c|}{ SURGERY TYPE } & \multirow[t]{2}{*}{ Total } \\
\hline & & BMR & $R / R$ & \\
\hline Range & $<35$ PD & 6 & 7 & 13 \\
\hline of pre-op & 35-50 PD & 8 & 20 & 28 \\
\hline PBCT & $>50$ PD & 19 & 19 & 38 \\
\hline Total & & 33 & 46 & 79 \\
\hline
\end{tabular}

PBCT- Prism Bar Cover Test; PD- Prism Diopter; BMR- Bimedial Recession; RR- Recession-Resection

\begin{tabular}{|c|c|c|c|c|}
\hline & & \multicolumn{2}{|c|}{ SURGERY TYPE } & \multirow[t]{2}{*}{ Total } \\
\hline & & BMR & $\mathbf{R} / \mathbf{R}$ & \\
\hline Post-op & $<35$ PD & 33 & 45 & 78 \\
\hline PBCT range & 35-50 PD & 0 & 1 & 1 \\
\hline Total & & 33 & 46 & 79 \\
\hline
\end{tabular}

PBCT- Prism Bar Cover Test; PD- Prism Diopter; BMR- Bimedial Recession; RR- Recession-Resection

of them had residual ET of $20 \mathrm{PD}$ and 1 had an angle of 30PD. In the RR group $7 / 46$ patients had angle $<35 \mathrm{PD}$, while 20/46 had angle between 35-50 PD, whereas 19/46 had angle $>50$ PD pre-operatively. Post-operatively during the second follow-up at third month $45 / 46$ patients were now observed to have angle $<35 \mathrm{PD}$, whereas $1 / 46$ patients had angle between 35-50 PD. Out of 45 patients who had angle between 35-50 PD, 33 of them had no angle, $1 / 45$ had 10 residual esotropia, whereas $3 / 45,5 / 45,1 / 45,2 / 45$, $1 / 45$ had 15, 20, 25, 30 and 35 PD of residual esotropia respectively. All patients who had residual ET in either the BMR or RR group had a pre-operative angle above 50 PD.

\section{DISCUSSION}

The majority of studies reported no effect of sex. ${ }^{6,7}$ In our study 38/79 (48.1\%) were male and 41/79 (51.9\%) were female. There was no effect of gender. Our study showed an overall mean age for presentation of infantile esotropia to range from 1-3 years which was seen in 34/79 (43\%) of cases; whereas $20 / 79$ cases $(25.3 \%$ ) presented between birth to 6 months, thus being the second most common age of presentation. The delayed presentation in my study compared to other studies where mean age of presentation was 6 months to 1 year of age was mainly due to lack of knowledge, poor cosmetic awareness and appreciation on parents side. Most patients who presented in my setting had their squint noticed at the age of schooling where the teachers reported about poor vision, inattentiveness in class and observed squinting which usually manifested during tasks requiring attentiveness and concentration.
In a study for binocularity following surgical correction of strabismus a retrospective study was conducted by Mets MB et al. which concluded that the benefits of surgical correction of strabismus in adults include improvement in binocular function, as seen in $42 \%$ of the patients in the study. ${ }^{8}$ The most common functional benefits from strabismus surgery in the adult patient are restoration of binocular vision and elimination of diplopia and/or a compensatory head posture. Adult patients with chronic strabismus have been thought to have the potential to achieve only a cosmetic result from surgery. On the contrary, several studies have shown that unexpected sensory fusion is possible after excellent postoperative motor alignment in these patients. Other functional benefits of strabismus surgery in the adult patient include the expansion of binocular visual fields in patients with esotropia and the psychosocial and economic benefits from restoration of ocular alignment. ${ }^{9}$ Binocular single vision was present in 50\% patients preoperatively and $53 \%$ patients postoperatively. Though cosmesis has been improved in most of the patients after strabismus surgery, binocular single vision and stereopsis improved in very minimal percentage of patients as the surgery was done after the age of visual maturation in most of the cases. This accentuates the need for strabismus surgery within the years of cortical plasticity. ${ }^{10}$ In our study level of BSV was assessed by Random dot test. Pre-operatively 43\% had fusion and rest of them had suppression. Post-operatively $40.5 \%$ had fusion, $15.2 \%$ had stereopsis and rest of them had suppression or BSV could not be assessed in them. Therefore there was increase in level of BSV by $2.5 \%$ from fusion to stereopsis. Those who initially showed suppression or in whom the BSV could not be assessed pre-operatively now showed to have fusion or some stereopsis. In total there was $15.2 \%$ increase in stereopsis. The level of binocular single vision (BSV) pre-operatively, it was seen that $17 / 33$ patients in the BMR group now had fusion, 8/33 did not have any BSV while in the rest $8 / 33$ the BSV could not be assessed. Post-operatively during the second follow up at third month 8/33 (24.24\%) developed stereopsis. It was seen that 4 out of the 17 candidates in the fusion group now developed stereopsis post-operatively during their second follow up at the third month. One of the candidates who did not have any level of BSV now developed stereopsis after surgery; while in 3 out of the 8 candidates in whom the BSV could not be assessed now showed to have stereopsis. In the RR group, pre-operatively 17/ 46 (36.95\%) had fusion, 28/46 did not have BSV, and in 1/46 BSV could not be assessed. Post-operatively during the second follow-up at third month $4 / 46(8.69 \%)$ of them in the RR group developed stereopsis, where as 2 additional candidates developed fusion, with a total of $19 / 46(41.30 \%)$ candidates in the fusion group post operatively compared to $17 / 46$ $(36.95 \%)$ in the preoperative period. In 5 of 28 candidates 
who had no BSV pre-operatively, now developed either fusion or stereopsis. Post-operatively during the second follow up at third month $8 / 33$ developed stereopsis. It was seen that 4 out of the 17 candidates in the fusion group now developed stereopsis post-operatively during their second follow up at the third month. One of the candidates who did not have any level of BSV now developed stereopsis after surgery; while in 3 out of the 8 candidates in whom the BSV could not be assessed now showed to have stereopsis. In the RR group, pre-operatively 17/46 had fusion, 28/46 did not have BSV, and in 1/46 BSV could not be assessed. Post-operatively during the second follow-up at third month 4 of them in the RR group developed stereopsis, where as 2 additional candidates developed fusion, with a total of 19 candidates in the fusion group post operatively compared to 17 in the preoperative period. In 5 of 28 candidates who had no BSV pre-operatively, now developed either fusion or stereopsis.

The age range in our study was divided into three groups: with age $<5$ years in group 1, between 5-10 years of age in group 2 and those above 10 years as group 3. The categorization was made in convenience with the age for visual maturity so that the outcomes of various intervention could be assessed. The mean age of surgery in our study was 10.11 years with most common age range for surgery being 10 years and above in $53.2 \%(42 / 79)$. It was seen that only $1 / 79(1.26 \%)$ of the candidates in group 1 attained BSV, whereas $7 / 79(8.8 \%)$ in group 2 age range developed stereopsis and 4/79 (5.06\%) developed stereopsis. Angle of deviation as well as history of optical therapy and patching should be taken into account for better outcome after the surgery as these nonsurgical modality of treatment aid in reducing deviation, maintaining alignment, fusion and preserving binocularity to some extent. In our study all those who attained stereopsis or had good alignment after surgery either had deviation $<50 \mathrm{PD}$, or had received some refractive correction or patching therapy. Those who did not attain BSV either had large deviation $>50$ PD or were amblyopic with no previous history of patching or refractive correction. Even those who developed binocularity had gross and not fine stereopsis. The optimal age for surgery for infantile esotropia is controversial.

The ELISSS compared early with late surgery in a prospective, controlled, non-randomized, multicenter trial. Children scheduled for early surgery had first been operated at 20 (SD 8.4) months, but 8.2\% had not been operated at age six. Children scheduled for late surgery had been operated at 49.1 (SD 12.7) months, but $20.1 \%$ had not been operated at age six. The number of operations per child was 1.18 (SD 0.67) in the early and 0.99 (SD 0.64) in the late group Children operated early had better gross stereopsis at age six as compared to children operated late. They had been operated more frequently, however, and a substantial number of children in both groups had not been operated at all. ${ }^{11}$ The Congenital Esotropia Observational Study has defined a clinical profile of infants who will benefit most from early surgery, and several other studies have shown that early surgery does not lead to adverse long-term effects. Clinicians now should consider offering early surgery to patients with large-angle, constant infantile esotropia at or before 10 months of age. ${ }^{12}$ Wright et al suggested the concept of "the time window of binocular development" as the period before irreversible damage occurs on visual function. ${ }^{2}$ They also suggested that optimum timing of surgery might be $2^{1 / 2}-3$ months of age to obtain a good binocular function. Chino recommends early surgery (before 4 months of ages) in congenital strabismus.' The range of age at surgery in our study was older, compared to other reports. There was no difference in the average timing of surgery in patients with or without stereopsis. We thus could not find a relation between the outcome of stereopsis and the timing of surgery similar to other reports. However, authors who believe that very early surgery is less advantageous do so because of the difficulties of accurate evaluation of the eye position and a high incidence of attaining spontaneous remission at an extremely early age. ${ }^{46}$ Most infants in whom stereopsis was attained in their study had satisfactory eye alignment during the follow up after surgery and at final examination. Maintenance of proper eye alignment should be checked regularly afterwards to achieve a desirable binocular visual function. ${ }^{13}$

A total of 33/79 (41.8\%) patients underwent BMR surgery. 6/33(18.18\%) patients had deviation <35 PD, $8 / 33(24.24 \%)$ had deviation between 35-50 PD, whereas 19/33 (57.57\%) had deviation > 50 PD. In this BMR group 8/33 (24.4\%) patients developed stereopsis, 13/33 (39.39\%) patients had fusion while the rest had suppression or the level of BSV could not be assessed. Another group consisted of 46/79 (58.2\%) patients who underwent RR surgery had deviation $<35 \mathrm{PD}$ in $7 / 46$ (15.2\%) patients, $20 / 46(43.47 \%)$ had deviation between 35-50 PD, whereas 19/46 (41.30\%) had deviation $>50$ PD. Following surgery 4/46 (8.69\%) developed stereopsis, 19/46 (41.30\%) had fusion while the rest had suppression. Thus in our study type of surgery did not determine the quality of vision. Following RR surgery $71.73 \%$ of patients had good alignment whereas $28.27 \%$ had residual angle ranging from 10-35 PD and were planned for another surgery. In the BMR group after surgery $84.84 \%$ had good alignment whereas $15.15 \%$ had residual angle ranging from 10-35 PD and was planned for second surgery. Kim E and Choi DG compared the surgical outcomes between bilateral medial rectus recession (BMR) and unilateral medial rectus recession-lateral rectus resection (RR) for infantile esotropia. ${ }^{14}$ The mean preoperative esodeviation at near was 44.1 prism diopters (PD) in BMR, and 40.2 PD in $\mathrm{RR}$. There was no statistically significant difference in angles of deviation between BMR and RR from postoperative day 
1 to final follow-up, respectively. Whereas until postoperative month 6 the surgical success rate did not significantly differ, from postoperative year 1 to final follow-up it was significantly higher in BMR than in RR. The final success rates were $80.70 \%$ and $56.52 \%$ for BMR and RR, respectively. The reoperation rate, correspondingly, was significantly lower for BMR (17.54\%) than for RR (60.78\%).There was no significant difference in the mean postoperative angle of deviation between BMR and RR for infantile esotropia. However, the final success rate was higher and the reoperation rate was lower for BMR than for RR. Likewise in our study there was no statistically significant correlation between preoperative deviation and type of surgery for infantile esotropia, neither was there any strong correlation between BSV outcome and type of surgery (BMR/RR).

\section{CONCLUSION}

Most common age at which squinting was first noticed was 1-3 years with most common presenting age being 10 years and above. Treatment of refractive error and amblyopia has to be given importance and conventional occlusion therapy is simple and cost effective treatment for amblyopia. Both bimedial recession and uniocular recession - resection works well as the initial procedure in attaining motor alignment within acceptable limits. Degree of alignment after surgery depends on the size of preoperative deviation. Early diagnosis and appropriate treatment before attainment of visual maturity or during the period of cortical plasticity is advocated for attaining binocular single vision and thus prevent amblyopia in cases of esotropias.

\section{REFERENCES}

1. Elliott $S$ and Shafiq $A$. Interventions for infantile esotropia. Cochrane Database Syst Rev. 2013; (7).

https://doi.org/10.1002/14651858.CD004917.pub3
2. Archer SM, Sondhi $\mathrm{N}$ and Helveston EM. Strabismus in infancy. Ophthalmology. 1989; 96(1):133-137. https://doi.org/10.1016/S0161-6420(89)32932-0

3. Alec M. Ansons and Davis H. Diagnosis and Management of Ocular Motility Disorders, 4th Edition. Wiley.com. [cited 2019 Feb 22].

4. Ing MR. Early surgical alignment for congenital esotropia. Trans Am Ophthalmol Soc. 1981; 79:625-663.

5. Scott $A B$. Botulinum toxin injection into extraocular muscles as an alternative to strabismus surgery. Ophthalmology. 1980; 87(10):1044-1049.

https://doi.org/10.1016/S0161-6420(80)35127-0

6. Bremer DL, Palmer EA, Fellows RR, Baker JD, Hardy RJ, Tung B, et al. Strabismus in Premature Infants in the First Year of Life. Arch Ophthalmol.1998; 116(3):329-333.

https://doi.org/10.1001/archopht.116.3.329

7. B G Mohney, J C Erie, D O Hodge and S J Jacobsen. Congenital esotropia in Olmsted County, Minnesota.

8. Mets MB, Beauchamp $C$ and Haldi BA. Binocularity following surgical correction of strabismus in adults. Trans Am Ophthalmol Soc.2003;101:201-207.

9. Edelman PM. Functional benefits of adult strabismus surgery. Am Orthopt J. 2010; 60:43-47.

https://doi.org/10.3368/aoj.60.1.43

10. Keenan JM and Willshaw HE. Outcome of strabismus surgery in congenital esotropia.

https://doi.org//10.1136/bjo.76.6.342

11. Simonsz HJ, Kolling $\mathrm{GH}$ and Unnebrink K. Department of Ophthalmology, Erasmus Medical Center, Rotterdamthe Netherlands, Final Report of the Early vs. Late Infantile Strabismus Surgery Study (ELISSS), a Controlled, Prospective, Multicenter Study. Research Gate. [cited 2019 Feb 23]. https://doi.org/10.1080/09273970500416594

12. Wong AMF. Timing of surgery for infantile esotropia: sensory and motor outcomes. Can J Ophthalmol. 2008; 43(6):643-651. https://doi.org/10.3129/i08-115

13. Shirabe $H$, Mori $Y$, Dogru $M$ and Yamamoto $M$. Early surgery for infantile esotropia. Br J Ophthalmol.2000; 84(5):536-538. https://doi.org/10.1136/bjo.84.5.536

14. Kim E and Choi DG. Outcomes after the surgery for acquired nonaccommodative esotropia. BMC Ophthalmol. 2017; 130(217).

https://doi.org/10.1186/s12886-017-0527-y

\footnotetext{
Authors Contribution:

AKG- Concept and design of the study, reviewed literature, preparation and revision of manuscript; KB- Reviewed literature and revision of manuscript; RDYCollection of data and revision of manuscript; SKC- Design and revision of manuscript

Work attributed to:

Lumbini Eye Institute and Research Center, Siddharthanagar-3, Bhairahawa, Nepal

Orcid ID:

Dr. Aman Kumar Gupta - 10 https://orcid.org/0000-0003-3064-2779

Dr. Kabindra Bajracharya - ittps://orcid.org/0000-0002-1742-9220

Dr. Roshan Dev Yadav - id https://orcid.org/0000-0002-8356-3436

Dr. Salma K.C. Rai - (『) http://orcid.org/0000-0001-5559-1512

Source of Support: None, Conflict of Interest: None declared.
} 